\title{
The DKP oscillator with a linear interaction in the cosmic string space-time
}

\author{
Mansoureh Hosseinpour ${ }^{1, \mathrm{a}}$, Hassan Hassanabadi ${ }^{1, \mathrm{~b}}$, Fabiano M. Andrade ${ }^{2, \mathrm{c}}$ \\ ${ }^{1}$ Faculty of Physics, Shahrood University of Technology, P.O. Box 3619995161-316, Shahrood, Iran \\ 2 Departamento de Matemática e Estatística, Universidade Estadual de Ponta Grossa, Ponta Grossa, Paraná 84030-900, Brazil
}

Received: 24 September 2017 / Accepted: 18 January 2018 / Published online: 31 January 2018

(C) The Author(s) 2018. This article is an open access publication

\begin{abstract}
We study the relativistic quantum dynamics of a DKP oscillator field subject to a linear interaction in cosmic string space-time in order to better understand the effects of gravitational fields produced by topological defects on the scalar field. We obtain the solution of DKP oscillator in the cosmic string background. Also, we solve it with an ansatz in the presence of a linear interaction. We obtain the wave functions and the energy levels of the relativistic field in that background.
\end{abstract}

\section{Introduction}

The Duffin-Kemmer-Petiau (DKP) equation is a linear wave equation that enables theoretical physicists to investigate both spin- 0 and spin- 1 fields with a single equation in the relativistic regime [1-4]. It is a direct generalization of the Dirac equation based on the so-called DKP algebra [5]. This algebra admits three irreducible representations more commonly utilised in physics: a one-dimensional trivial representation, a five-dimensional representation for spin- 0 fields, and a tendimensional representation that describes spin-1 fields [6]. The DKP equation is more applicable and much richer than the Klein-Gordon and Proca equations which describe spin-0 and spin-1 particles, respectively, therefore the DKP equation is rather more famous. However, the DKP equation has wide applications on different areas including meson spectroscopy, cosmology and nuclear-hadron interactions [7-9]. The DKP equation has been studied with various types of potentials for spin-0 and spin-1 particles [10-17]. The DKP theory has been applied to the quark confinement problem of quantum chromodynamics [18] and to covariant Hamiltonian dynamics [19]. The theory has also been studied in the casual

\footnotetext{
a e-mail: hosseinpour.mansoureh@gmail.com

be-mail: hha1349@gmail.com

c e-mail: fmandrade@uepg.br
}

approach [20,21], in the context of five-dimensional Galilean invariance [22] and in the scattering of the $\mathrm{K}+$ nucleus [23]. This equation has been successful in describing high-energy interactions of hadrons with nuclei and other branches of physics. The DKP equation has already been used to study deuteron-nucleus scattering [9], meson nuclear interaction [7] and a-nucleus elastic scattering [8]. It has also been used in deuteron-nucleus scattering [9].

On the other hand, the Dirac equation including a linear harmonic potential was initially studied by Ito et al. [24], Cook [25] and Ui et al. [26]. This system was latterly called by Moshinsky and Szczepaniak as Dirac oscillator [27]. More specifically, they suggested to substitute in the free Dirac equation the momentum operator $\vec{p} \rightarrow \vec{p}-i m \omega \beta \vec{r}$. Their construction lead to a system in which the spectrum is very similar to that of the non-relativistic harmonic oscillator [27]. Physically, it can be shown that the Dirac oscillator interaction is a physical system, which can be interpreted as the interaction of the anomalous magnetic moment with a linear electric field [28,29].

As a relativistic quantum mechanical system, the Dirac oscillator has been widely studied. Because it is an exactly solvable model, several investigations have been developed in the context of this theoretical framework in the last years. Although the Dirac oscillator is normally introduced within the context of many body theory, relativistic quantum mechanics and quantum chromodynamics (in particular as an interquark potential and also as the confining part of the phenomenological Cornell potential). The interest in this issue appears in different contexts such as quantum optics [30-32], supersymmetry [28,33,34], nuclear reactions [35], the hadronic spectrum using the two-body Dirac oscillator $[36,37]$ a new representation for its solutions using the Clifford algebra $[38,39]$, non-commutative space $[40,41]$, thermodynamic properties [42], Lie algebra symmetries [43], supersymmetric (non-relativistic) quantum mechanics [44] the super symmetric path integral formalism [45] the chiral 
phase transition in presence of a constant magnetic field [31], the relativistic Landau in the presence of external magnetic field [46] the Aharonov-Bohm effect [47,48] condensed matter physical phenomena and graphene [49]. The DKP oscillator is an analogous to Dirac oscillator [27].

The DKP oscillator is a kind of tensor coupling with a linear potential which leads to the harmonic oscillator problem in the weak-coupling limit. Also, a sort of vector DKP oscillator (non-minimal vector coupling with a linear potential) has been a topic of recent investigation [50-54]. Vector DKP oscillator is the name given to the system with a Lorentz vector coupling which exhibits an equally spaced energy spectrum in the weak-coupling limit. The name distinguishes it from the system called a DKP oscillator with Lorentz tensor couplings of Refs. [11,12,16,55-66]. By using an external linear potential, the DKP equation for spin-0 representation leads to the harmonic oscillator with a strong spin-orbit coupling of the Thomas form for vector bosons. The latter system is called the DKP oscillator.

The DKP oscillator in the $(1+2)$-dimensional noncommutative phase space for spin- 0 particles has been investigated in the work of Guo et al. [65]. Yang et al. studied the DKP oscillator with spin-0 in a three-dimensional non-commutative phase space [66]. A generalised bosonic oscillator within the minimal length quantum mechanics has been analysed in [62]. De Melo et al. released a higherdimensional formulation of Galilean covariance to consider the non-commutative DKP oscillator [67]. Falek and Merad presented both spin-0 and spin-1 DKP equations in noncommutative space in the $(1+3)$-dimensional case [64]. Recently, there has been an increasing interest on the socalled DKP oscillator $[11,12,16,50,55-66,68]$.

Relativistic systems embedded in a magnetic cosmic string background has inspired a great deal of research in last years. A cosmic string is a linear defect that changes the topology of the medium when viewed globally. The space-time around a cosmic string is locally flat but not globally. The theory of general relativity predicts that gravitation is manifested as curvature of space-time. This curvature is characterised by the Riemann tensor. There are connections between topological properties of the space and local physical laws. The nontrivial topology of space-time, as well as its curvature, leads to a number of interesting gravitational effects. For example, it has been known that the energy levels of an atom placed in a gravitational field will be shifted as a result of the interaction of the atom with space-time curvature. Therefore, we have to consider the topology of the space-time in order to describe completely the physics of system.

There are good theoretical reasons for believing that these exotic objects do exist, and reasonable prospects of detecting the existence of cosmic strings. The cosmic strings are sufficiently massive to have noticeable gravitational effects, therefore the gravitational field of a cosmic string is quite remarkable. A particle placed at rest around a straight, infinite, static cosmic string will not be attracted to it, due to the fact that there is no local gravity. The space-time around a cosmic string is locally flat but not globally. The general way to understand the interaction between relativistic quantum mechanical particles and gravity is to solve the general relativistic form of their wave equations. These solutions are valuable tools for examining and improving models and numerical methods for solving complicated physical problems. The external gravitational field due to a cosmic string may be approximately described by a commonly called conical geometry. The nontrivial topology of this space-time leads to a number of interesting measurable effects like, for example, particle self-force $[69,70]$ and gravitational lensing [71], as well as for production of highly energetic particles [7274].

In this work, we examine the relativistic quantum dynamics of the DKP oscillator in the presence of the linear interaction, on the curved space-time of a cosmic string. From the corresponding DKP equation, we analyse the influence of the topological defect on the equation of motion, the energy spectrum and the wave function. We stress, though, that we do not have the intention here to give answers to the interesting (and even hard) question of the detection of cosmic strings. In Sect. 2, we introduce the covariant DKP equation. In Sect. 3 we present the covariant DKP oscillator in cosmic string background and obtain the solution of DKP oscillator In Sect. 4, we present solution of DKP oscillator presence linear interaction. Finally, in the Sect. 5 we present our conclusions.

\section{Covariant form of the DKP equation in the cosmic string background}

The cosmic string space-time with an internal magnetic field in cylindrical coordinates is described by the line element (units such that $\hbar=c=1$ ) $[75,76]$

$\mathrm{d} s^{2}=-\mathrm{d} t^{2}+\mathrm{d} r^{2}+\alpha^{2} r^{2} \mathrm{~d} \varphi^{2}+\mathrm{d} z^{2}$,

with $-\infty<z<\infty, \rho \geq 0$ and $0 \leq \varphi \leq 2 \pi$. The angular parameter $\alpha$ runs in the interval $(0,1]$ is related to the linear mass density $\mu$ of the string as $\alpha=1-4 \mu$ and corresponds to a deficit angle $\gamma=2 \pi(1-\alpha)$. From the geometrical point of view, the metric in Eq. (1) describes a Minkowski space-time with a conical singularity.

Since we are working with curvilinear coordinates, it is convenient to treat the DKP equation by using the mathematical formulation of this theory in curved space-time background. This geometry possess a conical singularity represented by the curvature tensor $R_{r, \varphi}^{r, \varphi}=[(1-\alpha) / 4 \alpha] \delta_{2}(\vec{r})$, where $\delta_{2}(\vec{r})$ is a two-dimensional delta function. This 
behaviour of the curvature tensor is denominated conical singularity. The conical singularity gives rise to the curvature concentrated on the cosmic string axis, in all other places the curvature is null. This configuration defines a conical singularity.

Thus, we start by writing the DKP equation for a free boson in curved space-time [1-3]

$\left(i \beta^{\mu} \nabla_{\mu}-M\right) \psi=0$.

The covariant derivative in (2) is given by [77]

$\nabla_{\mu}=\partial_{\mu}-\Gamma_{\mu}(x)$,

where $\Gamma_{\mu}$ are the spinorial affine connections given by

$\Gamma_{\mu}=\frac{1}{2} \omega_{\mu a b}\left[\beta^{a}, \beta^{b}\right]$.

In terms of the Minkowski flat space-time coordinates, the algebra generated by the $\beta^{a}$ matrices has three irreducible representations: a ten-dimensional one that is related to spin1 particles, a five-dimensional one relevant for spin- 0 particles (i.e., spinless particles) and an one-dimensional one which is trivial. In the spin- 0 representation, $\beta^{a}$ are $5 \times 5$ matrices defined as

$\beta^{0}=\left(\begin{array}{ccccc}0 & 1 & 0 & 0 & 0 \\ 1 & 0 & 0 & 0 & 0 \\ 0 & 0 & 0 & 0 & 0 \\ 0 & 0 & 0 & 0 & 0 \\ 0 & 0 & 0 & 0 & 0\end{array}\right), \quad \beta^{1}=\left(\begin{array}{ccccc}0 & 0 & -1 & 0 & 0 \\ 0 & 0 & 0 & 0 & 0 \\ 1 & 0 & 0 & 0 & 0 \\ 0 & 0 & 0 & 0 & 0 \\ 0 & 0 & 0 & 0 & 0\end{array}\right)$,

$\beta^{2}=\left(\begin{array}{ccccc}0 & 0 & 0 & -1 & 0 \\ 0 & 0 & 0 & 0 & 0 \\ 0 & 0 & 0 & 0 & 0 \\ 1 & 0 & 0 & 0 & 0 \\ 0 & 0 & 0 & 0 & 0\end{array}\right), \quad \beta^{3}=\left(\begin{array}{ccccc}0 & 0 & 0 & 0 & -1 \\ 0 & 0 & 0 & 0 & 0 \\ 0 & 0 & 0 & 0 & 0 \\ 0 & 0 & 0 & 0 & 0 \\ 1 & 0 & 0 & 0 & 0\end{array}\right)$.

The $\omega_{\mu b}^{a}$ matrices represent the spin connection and are given by

$\omega_{\mu b}^{a}=e_{a}^{\rho} e_{v}^{b} \Gamma_{\mu \rho}^{v}-e_{a}^{v} \partial_{\mu} e_{v}^{b}$.

The only non-null components are

$\omega_{\varphi}^{12}=-\omega_{\varphi}^{21}=1-\alpha$.

We can build the local reference frame through a noncoordinate basis with $e_{\mu}^{\bar{a}}$ where $e_{\mu}^{\bar{a}}$ and $e_{\bar{a}}^{\mu}$ are transformation matrices. The components of the non-coordinate basis $e_{\mu}^{\bar{a}}$ are called tetrads or vierbeins; they form our local reference frame. With the line element given by Eq. (1), we can use tetrads $e_{\bar{a}}^{\mu}$ and $e_{\mu}^{\bar{a}}$ as follows: $e_{\bar{a}}^{\mu}=\left(\begin{array}{cccc}1 & 0 & 0 & 0 \\ 0 & \cos \varphi & \sin \varphi & 0 \\ 0 & \frac{-\sin \varphi}{\alpha r} & \frac{\cos \varphi}{\alpha r} & 0 \\ 0 & 0 & 0 & 1\end{array}\right), e_{\mu}^{\bar{a}}=\left(\begin{array}{cccc}1 & 0 & 0 & 0 \\ 0 & \cos \varphi & -\alpha r \sin \varphi & 0 \\ 0 & \sin \varphi & \alpha r \cos \varphi & 0 \\ 0 & 0 & 0 & 1\end{array}\right)$.

The vierbeins form our local reference frame that satisfy the orthonormality conditions

$e_{a}^{\mu}(x) e_{\nu}^{a}(x)=\delta_{\nu}^{\mu}$,

$e_{\mu}^{a}(x) e_{b}^{\mu}(x)=\delta_{b}^{a}$,

and they satisfy

$g_{\mu \nu}(x)=e_{\mu}^{(a)}(x) e_{\nu}^{(b)}(x) \eta_{a b}$.

The Kemmer matrices in curved space-time are related to their Minkowski counterparts via

$\beta^{\mu}(x)=e_{a}^{\mu} \beta^{a}$,

which leads us to their explicit form

$\beta^{t}=\beta^{0}$,

$\beta^{r}=\beta^{1}$,

$\beta^{\varphi}=-\omega \beta^{0}+\frac{1}{r \eta} \beta^{2}$,

$\beta^{z}=\beta^{3}$,

where $\beta^{\circ}, \beta^{r}, \beta^{\varphi}$ and $\beta^{z}$ are the general form of the Kemmer matrices in the cosmic string space-time.

\section{Solution of DKP oscillator in cosmic string background}

In this section, we concentrate our efforts on the interaction called DKP oscillator. For this external interaction we use the non-minimal substitution

$\partial_{r} \rightarrow \partial_{r}+M \omega r \eta^{0}$,

where $\omega$ is the oscillator frequency and $\eta^{0}=2\left(\beta^{0}\right)^{2}-$ 1. Considering only the radial component, with the nonminimal substitution one gets

$$
\begin{aligned}
& {\left[i \beta^{\circ} \partial_{t}+i \beta^{r}\left(\partial_{r}+M \omega r \eta^{0}\right)+i \beta^{\varphi}\left(\partial_{\varphi}-\Gamma_{\varphi}\right)\right.} \\
& \left.\quad+i \beta^{z} \partial_{z}-M\right] \Psi(t, r, \varphi, z)=0
\end{aligned}
$$

As the interaction is time-independent one can write the spinor as

$\Psi(t, r, \varphi, z)=\mathrm{e}^{-i\left(E t-m \varphi-k_{z} z\right)} \Psi(r)$, 
where $E$ is the energy of the scalar boson, $m$ is the angular momentum quantum number, $k_{z}$ is the eigenvalue for the momentum in the $z$ direction. By decomposing the fivecomponent spinor as $\Psi(r)=\left(\psi_{1}(r), \ldots, \psi_{5}(r)\right)^{T}$, the DKP equation for scalar bosons becomes (for compactness of the following equations, we momentarily drop the $r$ dependence in the spinor components)

$-m \sin \varphi \psi_{1}-\alpha r M \psi_{3}-i \alpha r \cos \varphi\left[M \omega r \psi_{1}+\psi_{1}^{\prime}\right]=0$, $m \cos \varphi \psi_{1}-\alpha r M \psi_{4}-i \alpha r \sin \varphi\left[M \omega r \psi_{1}+\psi_{1}^{\prime}\right]=0$,

$k_{z} \psi_{1}-M \psi_{5}=0$,

$E \psi_{1}-M \psi_{2}=0$,

$\alpha r\left[-M \psi_{1}+E \psi_{2}+k_{z} \psi_{5}+i \cos \varphi\left(1-\alpha+\alpha M \omega r^{2}\right) \psi_{3}\right.$

$\left.-i m \psi_{4}-\alpha r \psi_{3}^{\prime}\right]$

$+i \sin \varphi\left[i m \psi_{3}+\left(1-\alpha+\alpha M \omega r^{2}\right) \psi_{4}-r \alpha \psi_{4}^{\prime}\right]=0$. where

$\xi_{1}=M^{2} \omega^{2}$,

$\xi_{2}=-E^{2}-k_{z}^{2}+M^{2}+\frac{(2 \alpha-1)}{\alpha} M \omega$,

$\xi_{3}=\frac{m^{2}}{\alpha^{2}}$.

In this manner, the energy levels for the relativistic can be obtained from the following constraint [78]:

$$
\begin{array}{r}
\alpha_{2} n-(2 n+1) \alpha_{5}+(2 n+1)\left(\sqrt{\alpha_{9}}+\alpha_{3} \sqrt{\alpha_{8}}\right) \\
+n(n-1) \alpha_{3}+\alpha_{7}+2 \alpha_{3} \alpha_{8}+2 \sqrt{\alpha_{8} \alpha_{9}}=0
\end{array}
$$

where

$$
\begin{array}{lllll}
\alpha_{1}=\alpha-\frac{1}{2}, & \alpha_{2}=0, & \alpha_{3}=0, & \alpha_{4}=\frac{1}{2}\left(\frac{3}{2}-\alpha\right), & \alpha_{5}=0, \\
\alpha_{6}=\xi_{1}, & \alpha_{7}=-\xi_{2}, & \alpha_{8}=\alpha_{4}{ }^{2}+\xi_{3}, & \alpha_{9}=\xi_{1}, & \alpha_{10}=1+2 \sqrt{\alpha_{8}}, \\
\alpha_{11}=2 \sqrt{\alpha_{6}}, & \alpha_{12}=\alpha_{4}+\sqrt{\alpha_{8}}, & \alpha_{13}=-\sqrt{\alpha_{6} .} &
\end{array}
$$

By solving the above system of equations in favour of $\psi_{1}(r)$ we get

$$
\begin{aligned}
& \psi_{2}=\frac{E \psi_{1}}{M}, \\
& \psi_{3}=\frac{-m \sin \varphi \psi_{1}+i\left[-\alpha M \omega r^{2} \cos \varphi \psi_{1}-\alpha r \cos \varphi \psi_{1}^{\prime}\right]}{\alpha r M},
\end{aligned}
$$

$\psi_{4}=\frac{m \cos (\varphi) \psi_{1}+i\left[-\alpha M \omega r^{2} \sin \varphi \psi_{1}-r \alpha \sin \varphi \psi_{1}^{\prime}\right]}{\alpha r M}$,

$\psi_{5}=\frac{k_{z} \psi_{1}}{M}$

Combining these results we obtain an equation of motion for the first component of the DKP spinor,

$$
\begin{aligned}
& \psi_{1}^{\prime \prime}(r)+\frac{\alpha-1}{\alpha r} \psi_{1}^{\prime}(r) \\
& -\left(E^{2}-M^{2}+k_{z}^{2}-\frac{(2 \alpha-1) M \omega}{\alpha}+\frac{m^{2}}{\alpha^{2} r^{2}}+M^{2} \omega^{2} r^{2}\right) \\
& \psi_{1}(r)=0 .
\end{aligned}
$$

In order to solve the above equation, we employ the change of variable, $s=r^{2}$. Thus, we rewrite the radial equation (23) in the form

$$
\begin{aligned}
& \psi_{1}^{\prime \prime}(s)+\frac{(2 \alpha-1)}{2 \alpha s} \psi_{1}^{\prime}(s) \\
& +\frac{1}{4 s^{2}}\left(-\xi_{1} s^{2}+\xi_{2} s-\xi_{3}\right) \psi_{1}(s)=0,
\end{aligned}
$$

As the final step, it should be mentioned that the corresponding wave function is

$\psi_{1}(r)=N r^{2 \alpha_{12}} \mathrm{e}^{\alpha_{13} r^{2}} L_{n}^{\alpha_{10}-1}\left(\alpha_{11} r^{2}\right)$,

where $N$ is the normalization constant.

\section{Solution of the DKP oscillator in the presence of a linear interaction}

Let us now to analyse the situation when a DKP field interacts with a scalar potential $U(r)$, which is introduced via the substitution $M \rightarrow M+U(r)$. Thus, (18) becomes

$$
\begin{aligned}
& \left\{i \beta^{\circ} \partial_{t}+i \beta^{r}\left(\partial_{r}+M \omega r \eta^{0}\right)+i \beta^{\varphi}\left(\partial_{\varphi}\right.\right. \\
& \left.\left.-\Gamma_{\varphi}\right)+i \beta^{z} \partial_{z}-[M+U(r)]\right\} \Psi(t, r, \varphi, z)=0 .
\end{aligned}
$$

Here we are interested in studying the linear scalar potential:

$U(r)=a r$.

In order to solve Eq. (30) we make the following change of variables:

$\psi_{1}(r)=r^{\frac{1-\alpha}{2 \alpha}}(M+a r)^{\frac{1}{2}} R_{n, m}(r)$. 
This leads to an equation without first-order derivative term

$$
\begin{aligned}
& R_{n, m}^{\prime \prime}(r)+\left[-E^{2}-k_{z}^{2}+M\left(M+\omega-\frac{\omega}{\alpha}\right)\right. \\
& +\frac{\alpha^{2}-4 m^{2}-1}{4 \alpha^{2} r^{2}}-\frac{a(1-\alpha)}{2 M \alpha r}+2 a M r \\
& +\left(a^{2}-M^{2} \omega^{2}\right) r^{2}-\frac{3 a^{2}}{4(M+a r)^{2}} \\
& \left.+\frac{a^{2}(1-\alpha)+2 M^{3} \alpha \omega}{2 M \alpha(M+a r)}\right] R_{n, m}(r)=0 .
\end{aligned}
$$

The next step is to write $R_{n, m}(r)$ in the following form:

$$
R_{n, m}(r)=R_{n}(r) \mathrm{e}^{g_{m}(r)} .
$$

Thus, the $r$-dependent terms in Eq. (34) suggest that we take $g_{m}(r)$ as

$g_{m}(r)=b_{1} r+b_{2} r^{2}+b_{3} \log (r)+b_{4} \log (M+a r)$,

where the four constants $b_{1}, \ldots, b_{4}$ are to be expressed in terms of the physical constants $\alpha, a, \omega, M, m, k_{z}$ and $E$. For a general $n$ it is quite difficult to solve Eq. (33). However, we can be less ambitious and solve it for nodeless states, i.e., for $n=0$. In the latter case, we have $R_{n}(r)=1$; consequently [79-81]

$$
R_{0, m}(r)=\mathrm{e}^{g_{m}(r)} .
$$

In this manner, from Eq. (28) we have

$$
R_{0, m}^{\prime \prime}(r)+\left(-g_{m}^{\prime \prime}-g_{m}^{\prime 2}\right) R_{0, m}(r)=0 .
$$

Thus substituting Eq. (35) into Eq. (37), the latter equation becomes

$$
\begin{aligned}
& R^{\prime \prime}{ }_{0, m}(r)+\left(-b_{1}^{2}-2 b_{2}-4 r b_{1} b_{2}-4 b_{2} b_{3}\right. \\
& -4 b_{2} b_{4}-4 b_{2}^{2} r^{2}+\frac{b_{3}-b_{3}^{2}}{r^{2}} \\
& -\frac{2 M b_{1} b_{3}+2 a b_{3} b_{4}}{M r}+\frac{a^{2} b_{4}-a^{2} b_{4}^{2}}{(M+a r)^{2}} \\
& \left.+\frac{4 M^{2} b_{2} b_{4}-2 M a b_{1} b_{4}+2 a^{2} b_{3} b_{4}}{M(M+a r)}\right) R_{0, m}(r)=0 .
\end{aligned}
$$

If we compare Eq. (38) with Eq. (33), we have the following six equations:

$$
\begin{aligned}
& -E^{2}-k_{z}^{2}+M\left(M+\omega-\frac{\omega}{\alpha}\right)+b_{1}^{2}+2 b_{2}+4 r b_{1} b_{2} \\
& +4 b_{2} b_{4}+4 b_{2} b_{3}=0, \\
& -2 a b_{1} b_{4}+4 M b_{2} b_{4}+2 a^{2} b_{3} b_{4}+\frac{a^{2}(-1+\alpha)}{2 M \alpha}-M^{2} \omega=0,
\end{aligned}
$$

$\frac{2 a b_{3} b_{4}}{M}+2 b_{1} b_{3}+\frac{a(-1+\alpha)}{2 M \alpha}=0$,

$\frac{-1+\alpha^{2}-4 m^{2}}{4 \alpha^{2}}-b_{3}+b_{3}^{2}=0$,

$\left(b_{4}-b_{4}^{2}\right)+\frac{3}{4}=0$,

$4 b_{2}^{2}+\left(a^{2}-M^{2} \omega^{2}\right)=0$,

$4 b_{1} b_{2}+2 a M=0$.

These equations can be solved for $b_{1}, b_{2}, b_{3}$ and $b_{4}$. They also provide constraints on the physical parameters, in particular, o the energy $E$. Equation (39) admits the following solutions:

$b_{1}=\mp \frac{a M}{\sqrt{-a^{2}+M^{2} \omega^{2}}}$,

$b_{2}= \pm \frac{1}{2} \sqrt{-a^{2}+M^{2} \omega^{2}}$,

$b_{3}= \pm \frac{\alpha^{2}+\sqrt{\alpha^{2}+4 \alpha^{2} m^{2}}}{2 \alpha^{2}}$,

$b_{4}=\frac{1 \pm 2}{2}$.

and the energy levels are given by

$$
\begin{aligned}
E= & \pm\left[-k z^{2}+M^{2}+M \omega-\frac{M \omega}{\alpha}\right. \\
& \left.\left.+b_{1}^{2}+2 b_{2}+4 r b_{1} b_{2}+4 b_{2} b_{3}+4 b_{2} b_{4}\right]\right]^{1 / 2} .
\end{aligned}
$$

Finally, the nodeless wave function for DKP oscillator interacting with a linear scalar potential can be written as

$$
\begin{aligned}
\Psi_{0, m}(t, r, \varphi, z)= & N_{0, m} \mathrm{e}^{i\left(k_{z}+m \varphi-E t\right)} \mathrm{e}^{b_{1} r+b_{2} r^{2}} r^{b_{3}+\frac{1-\alpha}{2 \alpha}} \\
& \times(M+a r)^{b_{4}+\frac{1}{2}},
\end{aligned}
$$

where $N_{0, m}$ is normalization constant.

\section{Conclusion}

In this contribution, we have studied the relativistic quantum dynamics of a DKP oscillator field subject to a linear interaction for spin-0 particle in the cosmic string space-time. The DKP oscillator is a kind of tensor coupling with a linear potential which leads to the harmonic oscillator in the non-relativistic limit. This problem can be interpreted as an interaction of the anomalous magnetic moment with a linear electric field. From the corresponding DKP equation, we analyse the influence of the topological defect on the equation of motion, the energy spectrum and the wave function. We obtained a second order differential equation and proposed a new manner to solve it by changing the variable and using the NU method, leading to a solution in terms of the Laguerre polynomials. Our results are dependent on the 
angular parameter $\alpha$, which, by their turn, is related to the linear mass density of the cosmic string. In the limit case of $\alpha=1$, i.e., in the absence of a topological defect, we recover known results for the flat space-time. We also had considered the DKP oscillator in the presence of scalar interaction introduced by the substitution $M \rightarrow M+U(r)$. By choosing a suitable ansatz, the solution for this latter problem was obtained along with the energy spectrum. Here we have showed how the introduction of a topological defect, a cosmic string, leads to modifications in the spectrum and wave function of a spin- 0 particle.

Acknowledgements FMA was partially supported by the Brazilian agencies Conselho Nacional de Desenvolvimento Científico e Tecnológico $(\mathrm{CNPq})$ and Fundação Araucária. It is a pleasure for the authors to thank the referee for their many useful comments on the manuscript. The authors also thank Edilberto O. Silva for useful discussions. This study was funded by Conselho Nacional de Desenvolvimento Científico e Tecnológico.

Open Access This article is distributed under the terms of the Creative Commons Attribution 4.0 International License (http://creativecomm ons.org/licenses/by/4.0/), which permits unrestricted use, distribution, and reproduction in any medium, provided you give appropriate credit to the original author(s) and the source, provide a link to the Creative Commons license, and indicate if changes were made. Funded by SCOAP ${ }^{3}$.

\section{References}

1. G. Petiau, University of Paris thesis. Acad. R. Belg. Mem. Collect. 16(2), 1 (1936)

2. N. Kemmer, Quantum theory of Einstein-Bose particles and nuclear interaction. Proc. R. Soc. A 166(924), 127-153 (1938)

3. R.J. Duffin, On the characteristic matrices of covariant systems. Phys. Rev. 54(12), 1114-1114 (1938)

4. N. Kemmer, The particle aspect of meson theory. Proc. R. Soc. A 173(952), 91-116 (1939)

5. Walter Greiner, Relativistic Quantum Mechanics. Wave Equations (Springer, Berlin, 2000)

6. E.M. Corson, Introduction to Tensors, Spinors Relativistic Wave Equations (Chelsea Publication, Chelsea, 1953)

7. Roger C. Barrett, Youcef Nedjadi, Meson-nuclear interactions in the Duffin-Kemmer-Petiau formalism. Nucl. Phys. A 585(1-2), 311-312 (1995)

8. S. Ait-Tahar, J.S. Al-Khalili, Y. Nedjadi, A relativistic model for $\alpha$-nucleus elastic scattering. Nucl. Phys. A 589(2), 307-319 (1995)

9. R.E. Kozack, B.C. Clark, S. Hama, V.K. Mishra, R.L. Mercer, L. Ray, Spin-one Kemmer-Duffin-Petiau equations and intermediateenergy deuteron-nucleus scattering. Phys. Rev. C 40(5), 2181-2194 (1989)

10. A. Boumali, Particule de spin-1 dans un potentiel d'AharonovBohm. Can. J. Phys. 85(12), 1417-1429 (2007)

11. A. Boumali, On the eigensolutions of the one-dimensional DuffinKemmer-Petiau oscillator. J. Math. Phys. 49(2), 022302 (2008)

12. A. Boumali, One-dimensional thermal properties of the Kemmer oscillator. Phys. Scr. 76(6), 669-673 (2007)

13. H. Hassanabadi, B.H. Yazarloo, S. Zarrinkamar, A.A. Rajabi, Duffin-Kemmer-Petiau equation under a scalar Coulomb interaction. Phys. Rev. C 84(6), 064003 (2011)
14. S. Zarrinkamar, A.A. Rajabi, B.H. Yazarloo, H. Hassanabadi, An approximate solution of the DKP equation under the Hulthén vector potential. Chin. Phys. C 37(2), 023101 (2013)

15. S. Zarrinkamar, S .F. Forouhandeh, B .H. Yazarloo, H. Hassanabadi, Scattering states of the Duffin-Kemmer-Petiau equation for the Hulthén potential. Eur. Phys. J. Plus 128(9), 109 (2013)

16. Y. Kasri, L. Chetouani, Energy spectrum of the relativistic DuffinKemmer-Petiau equation. Int. J. Theor. Phys. 47(9), 2249-2258 (2008)

17. H. Hassanabadi, S.F. Forouhandeh, H. Rahimov, S. Zarrinkamar, B.H. Yazarloo, Duffin-Kemmer-Petiau equation under a scalar and vector Hulthen potential; an ansatz solution to the corresponding Heun equation. Can. J. Phys. 90(3), 299-304 (2012)

18. Vladimir Gribov, QCD at large and short distances (annotated version). Eur. Phys. J. C 10(1), 71-90 (1999)

19. Igor V. Kanatchikov, On the Duffin-Kemmer-Petiau formulation of the covariant hamiltonian dynamics in field theory. Rep. Math. Phys. 46(1-2), 107-112 (2000)

20. J.T. Lunardi, B.M. Pimentel, J.S. Valverde, L.A. Manzoni, DuffinKemmer-Petiau theory in the causal approach. Int. J. Mod. Phys. A 17(02), 205-227 (2002)

21. J.T. Lunardi, B.M. Pimentel, R.G. Teixeira, J.S. Valverde, Remarks on Duffin-Kemmer-Petiau theory and gauge invariance. Phys. Lett. A 268(3), 165-173 (2000)

22. M. de Montigny, F.C. Khanna, A.E. Santana, E.S. Santos, J.D.M. Vianna, Galilean covariance and the Duffin-Kemmer-Petiau equation. J. Phys. A 33(31), L273-L278 (2000)

23. L.K. Kerr, B.C. Clark, S. Hama, L. Ray, G.W. Hoffmann, Theoretical and experimental $\mathrm{k}^{+}+$nucleus total and reaction cross sections from the KDP-RIA model. Progr. Theor. Phys. 103(2), 321-335 (2000)

24. D. Itô, K. Mori, E. Carriere, An example of dynamical systems with linear trajectory. Nuovo Cim. A 51(4), 1119 (1967)

25. P.A. Cook, Relativistic harmonic oscillators with intrinsic spin structure. Lett. Nuovo Cim. 1(10), 419-426 (1971)

26. H. Ui, G. Takeda, Does accidental degeneracy imply a symmetry group? Progr. Theor. Exp. Phys. 72(2), 266-284 (1984)

27. M. Moshinsky, A. Szczepaniak, The Dirac oscillator. J. Phys. A 22(17), L817 (1989)

28. R P Martínez y Romero, A .L. Salas-Brito, Conformal invariance in a Dirac oscillator. J. Math. Phys. 33(5), 1831-1836 (1992)

29. M. Moreno, A. Zentella, Covariance, CPt and the FoldyWouthuysen transformation for the Dirac oscillator. J. Phys. A 22(17), L821 (1989)

30. D. Dutta, O. Panella, P. Roy, Pseudo-Hermitian generalized Dirac oscillators. Ann. Phys. (NY) 331, 120-126 (2013)

31. A. Bermudez, M .A. Martin-Delgado, A. Luis, Chirality quantum phase transition in the Dirac oscillator. Phys. Rev. A 77(6), 063815 (2008)

32. A. Bermudez, M.A. Martin-Delgado, E. Solano, Exact mapping of the $2+1$ Dirac oscillator onto the Jaynes-Cummings model: ion-trap experimental proposal. Phys. Rev. A 76(4), 041801 (2007)

33. J. Bentez, R.P.Martnez y Romero, H.N. Núez-Yépez, A.L. SalasBrito, Solution and hidden supersymmetry of a Dirac oscillator. Phys. Rev. Lett. 64(14), 1643-1645 (1990)

34. O. Castaños, A. Frank, R. López, L.F. Urrutia, Soluble extensions of the Dirac oscillator with exact and broken supersymmetry. Phys. Rev. D 43(2), 544-547 (1991)

35. J. Grineviciute, Dean Halderson, Dirac oscillators and the relativistic R matrix. Phys. Rev. C 80(4), 044607 (2009)

36. M. Moshinsky, Y.F. Smirnov, The Harmonic Oscillator in Modern Physics, Contemporary concepts in physics (Harwood Academic Publishers, Amsterdam, 1996)

37. M. Moshinsky, G. Loyola, Barut equation for the particleantiparticle system with a Dirac oscillator interaction. Found. Phys. 23(2), 197-210 (1993) 
38. R. de Lima Rodrigues, On the Dirac oscillator. Phys. Lett. A 372(15), 2587-2591 (2008)

39. James P. Crawford, The dirac oscillator and local automorphism invariance. J. Math. Phys. 34(10), 4428-4435 (1993)

40. F. Vega, Oscillators in a $(2+1)$-dimensional noncommutative space. J. Math. Phys. 55(3), 032105 (2014)

41. Shaohong Cai, Tao Jing, Guangjie Guo, Rukun Zhang, Dirac oscillator in noncommutative phase space. Int. J. Theor. Phys. 49(8), 1699-1705 (2010)

42. M.H. Pacheco, R.R. Landim, C.A.S. Almeida, One-dimensional Dirac oscillator in a thermal bath. Phys. Lett. A 311(2-3), 93-96 (2003)

43. C. Quesne, M. Moshinsky, Symmetry Lie algebra of the Dirac oscillator. J. Phys. A 23(12), 2263-2272 (1990)

44. J. Beckers, N. Debergh, Supersymmetry, Foldy-Wouthuysen transformations, and relativistic oscillators. Phys. Rev. D 42(4), 12551259 (1990)

45. R. Rekioua, T. Boudjedaa, Path integral for one-dimensional Dirac oscillator. Eur. Phys. J. C 49(4), 1091-1098 (2007)

46. Bhabani Prasad Mandal, Shweta Verma, Dirac oscillator in an external magnetic field. Phys. Lett. A 374(8), 1021-1023 (2010)

47. N. Ferkous, A. Bounames, Energy spectrum of a 2D Dirac oscillator in the presence of the Aharonov-Bohm effect. Phys. Lett. A 325(1), 21-29 (2004)

48. Fabiano M. Andrade, Edilberto O. Silva, Effects of spin on the dynamics of the 2D Dirac oscillator in the magnetic cosmic string background. Eur. Phys. J. C 74, 3187 (2014)

49. Emerson Sadurni, The Dirac-Moshinsky oscillator: theory and applications. AIP Conf. Proc. 1334(1), 249-290 (2011)

50. H. Hassanabadi, Z. Molaee, S. Zarrinkamar, DKP oscillator in the presence of magnetic field in $(1+2)$-dimensions for spin-zero and spin-one particles in noncommutative phase space. Eur. Phys. J. C 72(11), 2217 (2012)

51. D.A. Kulikov, R.S. Tutik, A.P. Yaroshenko, An alternative model for the Duffin-Kemmer-Petiau oscillator. Mod. Phys. Lett. A 20(01), 43-49 (2005)

52. T.R. Cardoso, L.B. Castro, A.S. de Castro, On the nonminimal vector coupling in the Duffin-Kemmer-Petiau theory and the confinement of massive bosons by a linear potential. J. Phys. A 43(5), $055306(2010)$

53. T.R. Cardoso, L.B. Castro, A.S. de Castro, Confining solutions of massive spin-0 bosons by a linear nonminimal vector coupling in the Duffin-Kemmer-Petiau theory. Nucl. Phys. B Proc. Suppl. 199(1), 203-206 (2010)

54. L.B. Castro, A.S. de Castro, Spinless bosons embedded in a vector Duffin-Kemmer-Petiau oscillator. Phys. Lett. A 375(27), 2596$2600(2011)$

55. N. Debergh, J. Ndimubandi, D. Strivay, On relativistic scalar and vector mesons with harmonic oscillator like interactions. Z. Phys. C Part. Fields 56(3), 421-425 (1992)

56. Y. Nedjadi, R .C. Barrett, The Duffin-Kemmer-Petiau oscillator. J. Phys. A 27(12), 4301-4315 (1994)

57. Y. Nedjadi, S. Ait-Tahar, R.C. Barrett, An extended relativistic quantum oscillator for particles. J. Phys. A 31(16), 3867-3874 (1998)

58. Y. Nedjadi, R.C. Barrett, A generalized Duffin-Kemmer-Petiau oscillator. J. Phys. A 31(31), 6717-6724 (1998)

59. A. Boumali, L. Chetouani, Exact solutions of the Kemmer equation for a Dirac oscillator. Phys. Lett. A 346(4), 261-268 (2005)
60. I. Boztosun, M. Karakoc, F. Yasuk, A. Durmus, Asymptotic iteration method solutions to the relativistic Duffin-Kemmer-Petiau equation. J. Math. Phys. 47(6), 062301 (2006)

61. F. Yasuk, M. Karakoc, I. Boztosun, The relativistic DuffinKemmer-Petiau sextic oscillator. Phys. Scr. 78(4), 045010 (2008)

62. M. Falek, M. Merad, A generalized bosonic oscillator in the presence of a minimal length. J. Math. Phys. 51(3), 033516 (2010)

63. M. Falek, M. Merad, Bosonic oscillator in the presence of minimal length. J. Math. Phys. 50(2), 023508 (2009)

64. M. Falek, M. Merad, DKP oscillator in a noncommutative space. Commun. Theor. Phys. 50(3), 587-592 (2008)

65. Guangjie Guo, Chaoyun Long, Zuhua Yang, Shuijie Qin, DKP oscillator in noncommutative phase space. Can. J. Phys. 87(9), 989-993 (2009)

66. Zu-Hua Yang, Chao-Yun Long, Shuei-Jie Qin, Zheng-Wen Long, DKP oscillator with spin-0 in three-dimensional noncommutative phase space. Int. J. Theor. Phys. 49(3), 644-651 (2010)

67. G.R. de Melo, M. de Montigny, E.S. Santos, Spinless DuffinKemmer-Petiau oscillator in a galilean non-commutative phase space. J. Phys. Conf. Ser. 343, 012028 (2012)

68. Luis B. Castro, Quantum dynamics of scalar bosons in a cosmic string background. Eur. Phys. J. C 75(6), 287 (2015)

69. E.R.Bezerra de Mello, V.B. Bezerra, YuV Grats, Self-forces in the spacetime of multiple cosmic strings. Class. Quantum Grav. 15(7), 1915-1925 (1998)

70. C.R. Muniz, V.B. Bezerra, Self-force on an electric dipole in the spacetime of a cosmic string. Ann. Phys. (NY) 340(1), 87-93 (2014)

71. M.V. Sazhin, O.S. Khovanskaya, M. Capaccioli, G. Longo, M. Paolillo, G. Covone, N.A. Grogin, E.J. Schreier, Gravitational lensing by cosmic strings: what we learn from the CSL-1 case. Mon. Not. R. Astron. Soc. 376(4), 1731-1739 (2007)

72. V.B. Bezerra, V.M. Mostepanenko, R.M.Teixeira Filho, Particle creation in the chiral cosmic string spacetime. Int. J. Mod. Phys. D 11(03), 437-445 (2002)

73. V.A. De Lorenci, R.D.M. De Paola, N.F. Svaiter, From spinning to non-spinning cosmic string spacetime. Class. Quantum Gravity 16(10), 3047-3055 (1999)

74. Jürgen Audretsch, Athanasios Economou, Quantum-fieldtheoretical processes near cosmic strings: transition probabilities and localization. Phys. Rev. D 44(4), 980-990 (1991)

75. Alexander Vilenkin, Cosmic strings and domain walls. Phys. Rep. 121(5), 263-315 (1985)

76. B. Linet, The static metrics with cylindrical symmetry describing a model of cosmic strings. Gen. Relat. Gravit. 17(11), 1109-1115 (1985)

77. A. Havare, T. Yetkin, K. Sogut, On the equivalence of the massless DKP equation and Maxwell equations in Robertson-Walker spacetime. Chin. J. Phys. 5, 465-474 (2003)

78. C. Tezcan, R. Sever, Dirac equation with vector and scalar Cornell potentials and an external magnetic field. Int. J. Theor. Phys 48, 337-350 (2009)

79. Timothy Clifton, John D. Barrow, The existence of Gödel, Einstein, and de Sitter universes. Phys. Rev. D 72(12), 123003 (2005)

80. Reinaldo J. Gleiser, Metin Gürses, Atalay Karasu, Özgür Sarinodotoğlu, Closed timelike curves and geodesics of Gödel-type metrics. Class. Quantum Gravity 23(7), 2653-2663 (2006)

81. Troels Harmark, Tadashi Takayanagi, Supersymmetric Gödel universes in string theory. Nucl. Phys. B 662(1-2), 3-39 (2003) 